Submitted to Astrophysical Journal

Preprint typeset using $\mathrm{IAT}_{\mathrm{E}} \mathrm{X}$ style emulateapj v. 12/16/11

\title{
EXCESSIVE SHIFT OF THE CMB ACOUSTIC PEAKS OF THE COLD SPOT AREA
}

\author{
Lung-YiH CHIANG \\ Institute of Astronomy and Astrophysics, Academia Sinica, 11F of Astro-Maths Building, AS/NTU. No.1, Sec.4, Roosevelt Rd, Taipei \\ 10617, Taiwan \\ Submitted to Astrophysical Journal
}

\begin{abstract}
Measurement of the acoustic peaks of the cosmic microwave background (CMB) temperature anisotropies has been instrumental in deciding the geometry and content of the universe. Acoustic peak positions vary in different parts of the sky owing to statistical fluctuation. We present the statistics of the peak positions of small patches from ESA Planck data. We found that the peak positions have significantly high variance compared to the $100 \mathrm{CMB}$ simulations with best-fit $\Lambda \mathrm{CDM}$ model with lensing and Doppler boosting effects included. Examining individual patches, we found the one containing the mysterious "Cold Spot", an area near the Eridanus constellation where the temperature is significantly lower than Gaussian theory predicts, displays large synchronous shift of peak positions towards smaller multipole numbers with significance lower than $1.11 \times 10^{-4}$. The combination of large synchronous shifts in acoustic peaks and lower than usual temperature at the Cold Spot area results in a $4.73 \sigma$ detection (significance $p \simeq 1.11 \times 10^{-6}$ ) against the $\Lambda$ CDM model. And it was already reported in Finelli et al. (2016) that in the WISE-2MASS galaxy catalog at $z<0.3$ the Cold Spot region is surrounded by surprisingly large underdense regions around $15^{\circ}$ in radius, which are found to be in the same square patch. Thus we propose there is some extra localized unknown energy to stretch out the space in the transverse direction around the Cold Spot area to simultaneously account for the Cold Spot, the excessive shift of the acoustic peaks, and the large underdense regions.
\end{abstract}

Keywords: cosmic microwave background — cosmology: observations — methods: data analysis

\section{INTRODUCTION}

The measurement of the acoustic peak positions of the cosmic microwave background (CMB) temperature anisotropies has been instrumental in deciding the geometry and content of the universe(Hu \& Dodelson 2002; Hinshaw et al. 2013), e.g. the first acoustic peak at $\ell \simeq 220$ indicating a flat universe. It was the Boomerang balloon experiment in 2000 that firstly shows the first acoustic peak position (in a partial sky) is measured at $\ell=197 \pm 6$ (de Bernardis et al. 2000), later MAXIMA-1 at $\ell \simeq 220$ (Hanany et al. 2000), and DASI at around 200 (Halverson et al. 2002), VSA at 224 (Scott et al. 1996), compared to full-sky WMAP measurement at $220.8 \pm 0.7$ (Hinshaw et al. 2007) and the latest Planck result to be $\ell=220.0 \pm 0.5$ (Planck Collaboration: Ade et al. 2014a; Planck Collaboration: Aghanim et al. 2016). The discrepancy is mainly due to the fact that the acoustic peak positions vary in different parts of the sky. In this paper, we test the statistics of the peak positions from ESA Planck data. This paper is arranged as follows. In Sec.2 we describe the data processing of Planck data and we use variance as the statistics on peak positions in Sec.3. We show the excessive peak shifts near the Cold Spot area in Sec.4. Discussion is in Sec.5.

\section{PLANCK DATA PROCESSING AND FITTING}

To test the statistics of the acoustic peaks, we use flat sky approximation (FSA) and take patches of $20 \times 20$ $\mathrm{deg}^{2}$ from the 4 Planck CMB maps: SMICA, NILC, SEVEM and Commander maps in GLESP pixelization(Doroshkevich et al. 2005), each with a specific fore-

lychiang@asiaa.sinica.edu.tw ground cleaning method on either harmonic or pixel domain (Planck Collaboration: Ade et al. 2014b). The map is rotated every $22^{\circ} .5$ (in practice it's the $a_{\ell m}$ of the map for rotation before being repixelized with GLESP with 2 arcmin/pixel) and we take the patches parallel to the $\theta$ and $\phi$ coordinate lines centered at the equator to minimize the error from pixelization. As such we separate the whole sphere into 90 patches. In our analysis we further use the Planck GAL070 mask (Planck Collaboration: Adam et al. 2016b) to block areas of heavy foreground contamination around Galactic plane, thus in total there are 45 patches available for test in each map, accounting for $43 \%$ of the whole sky.

Each patch is firstly interpolated in the $\theta$ direction as the spacing of the grid is slightly uneven due to the fact that the grids are where the Gauss-Legendre polynomial zeros are (Doroshkevich et al. 2005). Two-dimensional Fourier transform is used to analyze each patch. The scaling relation between integer Fourier wavenumber $k$ and multipole number $\ell$ is $\ell=2 \pi k / L$, where $L$ is the size of the patch. The amplitude of the angular power spectrum $S_{\ell}$ can be scaled from $S_{k}$ at Fourier wavenumber $k$ via

$$
S_{\ell \equiv 2 \pi k / L}=L^{2} S_{k}
$$

So for each patch with size $L$, the wavenumber $k$ is scaled into $2 \pi k / L$, and there is no binning in our power spectrum and the sequence of $k$ is scaled such that the multipole interval $\Delta \ell \equiv 2 \pi / L$. One can also see the choice of the patch size cannot be arbitrary. The sampling interval $\Delta \ell$ of angular power spectrum is inversely proportional to $L$, so we want to choose patches as large as possible to have more sampled data in the power spectrum, but it 

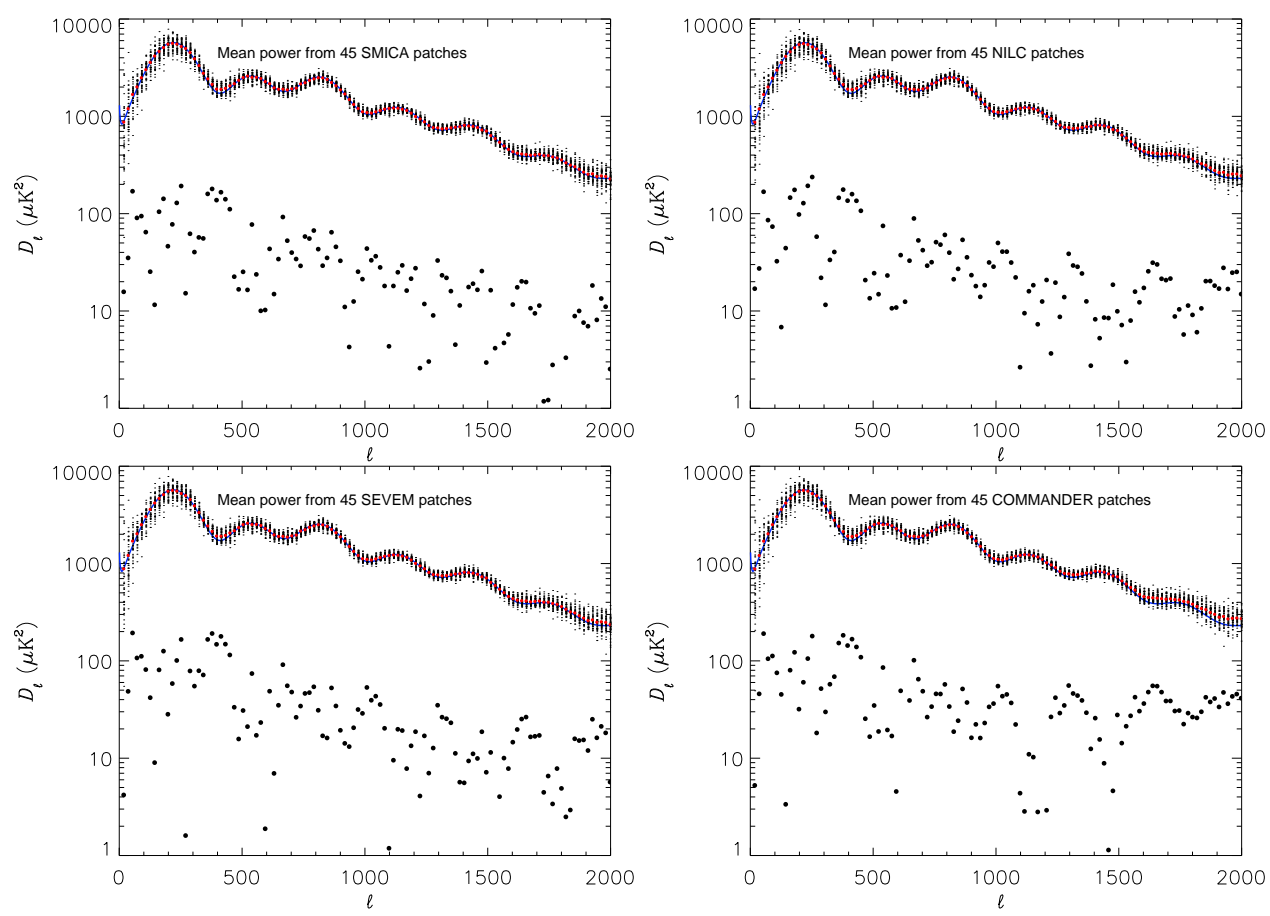

Figure 1. The angular power spectra from 45 patches of the Planck 4 maps. Top part in each panel is the mean power spectrum (big red dot) from the 45 patches (small black dot) and the Planck best-fit $\Lambda$ CDM power spectrum (blue curve), whereas the bottom part (big black dot) is the absolute difference between the mean and the Planck best-fit spectrum.

can't be too large as the curvature of the sphere causes the flat sky approximation to break down. The scaling relation from Fourier to spherical harmonic analysis is already tested with simulations (Chiang \& Chen 2012).

One issue related to Fourier transform on real signals is the non-periodic boundary (NPB) condition, which induces extra power from jump discontinuity. The condition is aggravated when there is beam convolution causing jump discontinuity more pronounced at the boundary. We have conducted simulations to estimate the extra power due to 5 -arcmin beam convolution on a $20 \times 20$ $\operatorname{deg}^{2}$ patch to be $1.21 \ell^{-1.38}\left(\mu \mathrm{K}^{2}\right)$ (see the Appendix A). This should be subtracted before any data processing.

It is assumed that the 4 Planck maps are fairly cleaned such that the angular power spectrum in each patch $S_{k}=b_{k}^{2} C_{k}+N_{k}$, where $C_{k}$ denotes the CMB, $b_{k}$ the beam transfer function and $N_{k}$ the noise. We then use cross-power spectrum (XPS) to reduce the noise (see Appendix B). The XPS is a quadratic estimator between two patches or maps to provide an unbiased estimate of the power spectrum of the correlated signals:

$$
b_{k}^{2} C_{k}^{\alpha \beta}=\frac{1}{2}\left\langle\alpha_{\boldsymbol{k}^{\beta}}^{\beta} \boldsymbol{k}+\beta_{\boldsymbol{k}}^{*} \alpha \boldsymbol{k}\right\rangle
$$

where $\alpha_{\boldsymbol{k}}$ and $\beta_{\boldsymbol{k}}$ are the Fourier modes of patches from Planck halfring maps, $*$ denotes complex conjugate and the angle brackets denote an average over all $k-1 / 2 \leq$ $|\boldsymbol{k}|<k+1 / 2$ for integer $\mathrm{k}$. The residual of the XPS $X_{k}^{\alpha \beta}$ has to be further subtracted, which stems from the lack of an ensemble and non-zero chance correlation between the "uncorrelated" signal (Chiang \& Chen 2011, 2012),

$$
\sqrt{\left\langle\left(X_{k}^{\alpha \beta}\right)^{2}\right\rangle} \simeq \frac{\sqrt{N_{k}^{\alpha} N_{k}^{\beta}}}{\sqrt{2 \pi k}}
$$

where $N_{k}^{\alpha}$ and $N_{k}^{\beta}$ are the (uncorrelated) noise power spectrum from halfring patches, respectively. The noise power spectrum can be obtain by $1 / 2$ of the differencing of halfring patches, assuming both are of the same level. Thus in our data pipeline, we subtract the residual $X_{k}^{\alpha \beta}$ from the XPS, following the correction of NPB.

It is then subsequently de-convolved with a beam transfer function at 5 arcmin (Planck Collaboration: Adam et al. 2016b). To check the validity of our data processing, in the 4 panels of Figure 1 we show the mean angular power spectrum from the 45 patches of the Planck 4 maps, which fits very well with the best-fit $\Lambda \mathrm{CDM}$ power spectrum. And in Fig 2 we show the dispersions of the 45 power spectra from the Planck patches, which fall within the range of the simulation up to $\ell=1,350$.

We use the Levenberg-Marquardt algorithm (Press et al. 1994) as the non-linear least-square fitting routine to fit the angular power spectrum curve with sum of 5 Gaussian functions to the beginning of the 5th peak (from $\ell=50$ to 1,350$)$. The peak positions are estimated in $\ell$ and only the first 3 peaks are estimated because the low amplitudes of the 4th and 5th peaks are prone to error in the estimation. The distribution of the peak positions of the SMICA map is represented in 3 dimensions of $\ell \equiv\left(\ell^{(1)}, \ell^{(2)}, \ell^{(3)}\right)$ in top panel of Figure 3 .

Any statistics from the data are to be compared with lensing simulations by Planck Consortium (Planck Collaboration: Ade et al. 2014c). There are 100 realizations based on best-fit $\Lambda \mathrm{CDM}$ model with both the lensing and the Doppler boosting effects included ${ }^{1}$, which are originally employed to determine the lensing mean field, the measurement error bars, and to validate lensing reconstruction methodology. The angular resolution

\footnotetext{
${ }^{1}$ http://wiki.cosmos.esa.int/planckpla/index.php/Simulation_data
} 


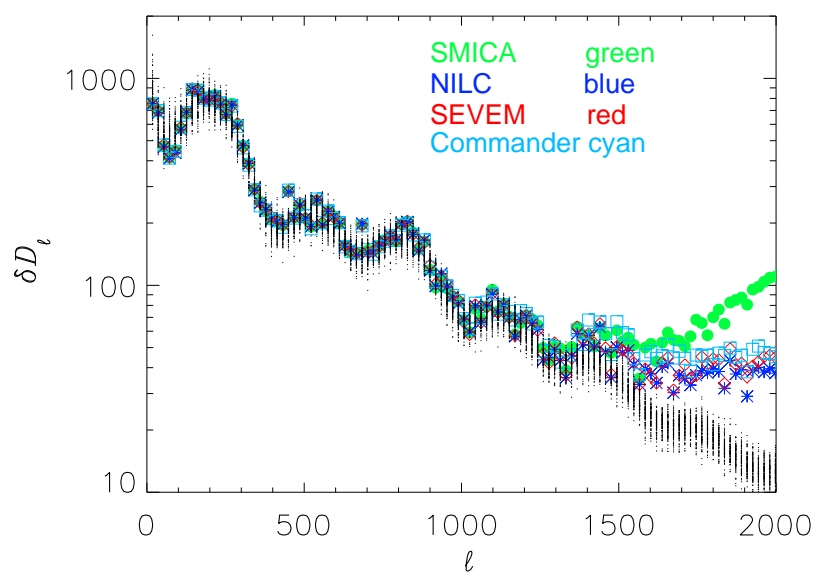

Figure 2. The dispersion of the 45 angular power spectra of the Planck patches with green filled circle, blue asterisk, red diamond and and light blue square sign denoting SMICA, NILC, SEVEM and Commander patches, respectively and those from the 100 simulations (black dot). One can see the dispersions from the Planck 4 maps fall within the 100 simulations up to $\ell=1,350$, which is then adopted as the the upper limit of our Gaussian-fitting range.

of the simulations is 5 arcmin, the same as the 4 Planck maps. Gravitational lensing effect (Blanchard \& Schneider 1987; Cole \& Efstathiou 1989; Linder 1990; Seljak 1996; Challinor \& Lewis 2005; Lewis \& Challinor 2006) is the deflection of CMB photons coming from the last scattering surface by potential gradients along our line of sight, and the Planck team used both temperature and polarization data to measure the lensing potential at a level of $40 \sigma$ (Planck Collaboration: Ade et al. 2014c, 2016a). The Doppler boosting effect (Challinor \& Lewis 2002), on the other hand, is due to the motion of our solar system barycenter with respect to the CMB rest frame that induces not only the dipolar anisotropy, but also the Doppler modulation and aberration at high multipoles (Planck Collaboration: Aghanim et al. 2014). Both effects on the angular power spectrum at high multipoles are small, but they can affect significantly the angular power spectrum of a small patch (Jeong et al. 2014). Since both effects are still in the Planck 4 maps, it is thus appropriate to take for comparison the simulation with the aforementioned effects included. We process the 100 simulations with the same pipeline as the Planck 4 maps with the same choice of patches and rescaling.

\section{VARIANCE OF THE PEAK POSITIONS}

We use the variance of the first 3 peaks to characterize the statistics of peak positions:

$$
\sum_{i=1}^{n}\left|\ell_{i}-\bar{\ell}\right|^{2} /(n-1)
$$

where $\bar{\ell}$ is the mean of the peak positions from the $n=45$ patches. In bottom panel of Figure 3 we plot the histogram of the variance from $100 \mathrm{CMB}$ realizations and those of the 4 Planck maps are denoted with colored lines. The result shows that the variances of the peak positions from 4 maps are all quite large with significance $p<0.04$, in particular the variance of the SMICA map is higher than 98 realizations, which is equivalent roughly to a significance $p$-value 0.02 against the null hypothesis.

We examine the possible effect from foreground resid-
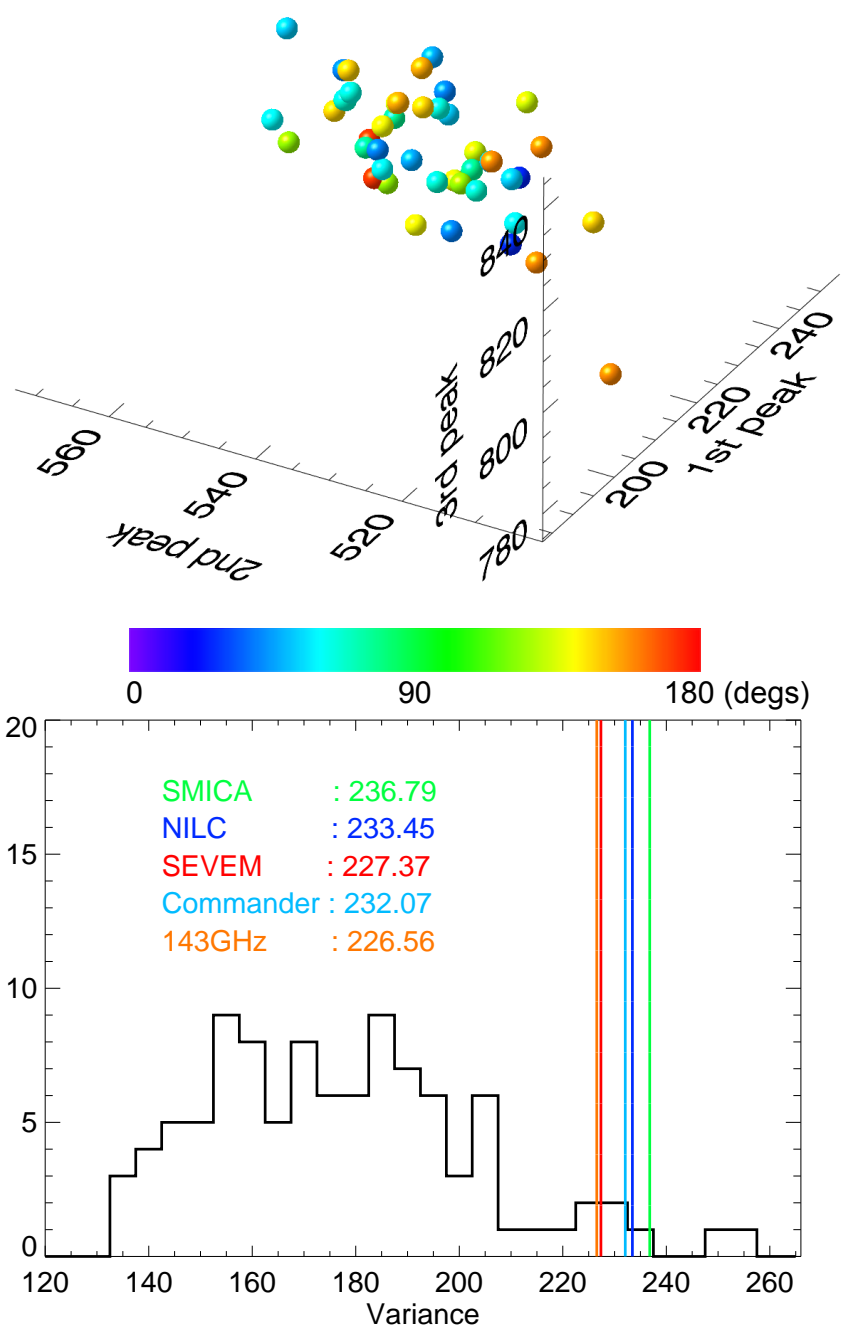

Figure 3. Distribution of the acoustic peak positions of the SMICA 45 patches and histogram of the variances of the peak positions from 100 realizations of Planck simulation. In the top panel each patch is represented with one point $\ell \equiv\left(\ell^{(1)}, \ell^{(2)}, \ell^{(3)}\right)$ with the peak positions denoted in 3 coordinates. The color in each point denotes the angle between the Galactic North Pole and the patch center. In the bottom the variances of the Planck 4 maps and $143 \mathrm{GHz}$ band map are denoted with colored lines.

ual with the mean and the dispersion of the 45 power spectra from the patches. It is based on the fact that any extra non-correlated signal added to the CMB would increase both the mean and dispersion of the power spectra. As shown in Figure 1 the mean angular power spectra fit well with the best-fit $\Lambda$ CDM power spectrum, and in Figure 2 the dispersions from the 45 patches fall within the 100 realizations up to $\ell=1,350$. Thus we are guaranteed that the data is at least free from significant foreground residual up to $\ell=1,350$.

If foreground residual is the cause of the anomaly, the variance from any frequency band map should be higher than that of the SMICA map because the foreground contamination in any band map is definitely higher than that in SMICA map. However, the variance for the Planck $143 \mathrm{GHz}$ channel map containing diffuse foreground is 
226.56 (where we only subtract bright point sources), smaller than that of SMICA map 236.79 instead (where the distance between the mean peak positions of $143 \mathrm{GHz}$ and those of SMICA is only 2.19). The foreground residual as the cause for the anomaly can then be ruled out. The influence of the Galactic plane is also tested by colorcoding the points for the distribution of the peaks. There is no obvious clustering in color, hence no systematic effect from either Galactic (as shown in top panel of Figure 3) or Ecliptic influence.

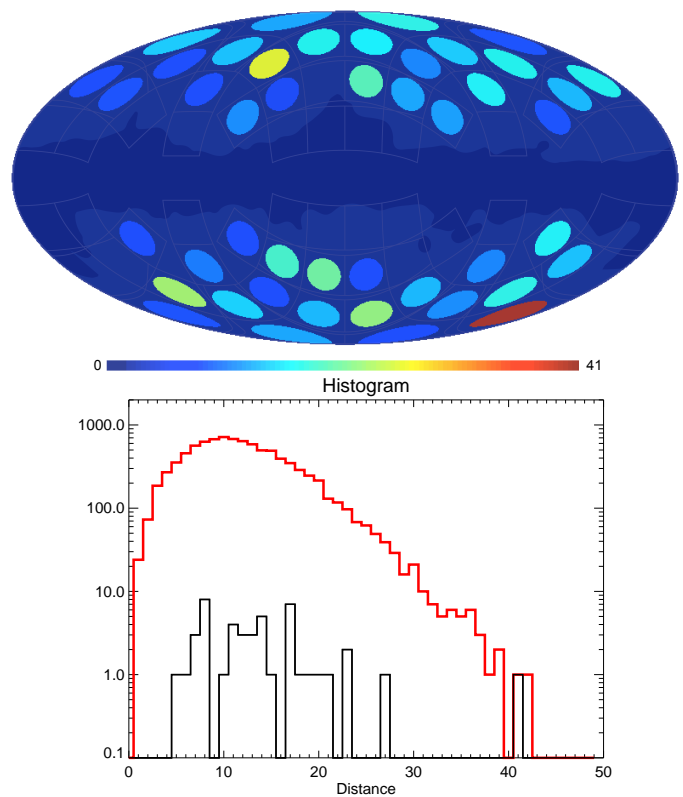

Figure 4. The peak distance from the mean: $\left|\ell_{i}-\bar{\ell}\right|$ of the 45 patches on the sky (top), and their histogram (black curve in the bottom panel). In the top panel we use large round symbol centered in each patch to denote the distance with color, and the patch with red color is centered at $(l, b)=\left(204^{\circ} .74,-65^{\circ} .48\right)$, where the famed Cold Spot is inside. In the bottom panel the red curve is the histogram of the 9000 patches from the Planck simulation.

\section{EXCESSIVE PEAK SHIFTS IN THE COLD SPOT PATCH}

Since the variance is related to the "distance" of the peak positions of patch $i$ to their mean, $\left|\ell_{i}-\bar{\ell}\right| \equiv$ $\sqrt{\left(\ell_{i}^{(1)}-\bar{\ell}^{(1)}\right)^{2}+\left(\ell_{i}^{(2)}-\bar{\ell}^{(2)}\right)^{2}+\left(\ell_{i}^{(3)}-\bar{\ell}^{(3)}\right)^{2}}$, we show the distance of the 45 patches on the sky in top panel of Figure 4. One can easily notice one patch has far larger distance 40.72 than the rest. That patch is centered at $(l, b)=\left(204^{\circ} .74,-65^{\circ} .48\right)$ near the Eridanus constellation, which contains the well-known CMB "Cold Spot" (CS) (Vielva et al. 2004; Cruz et al. 2005) (see top left panel of Figure 5), an area where the CMB temperature has lower than Gaussian theory predicts with significance around 1\% (Planck Collaboration: Ade et al. 2016b). One should also note that the 3 peaks of the patch with CS not only have larger shifts than usual, but also display synchronous shifts towards smaller multipole numbers (i.e. larger scales) than the mean, as shown in middle panel of Figure 5.

One should note that a decrement, in particular, an extended decrement in CMB temperature does not cause peak shifts, i.e. there is no correlation between a decrement in $\mathrm{CMB}$ temperature and peaks leaning towards
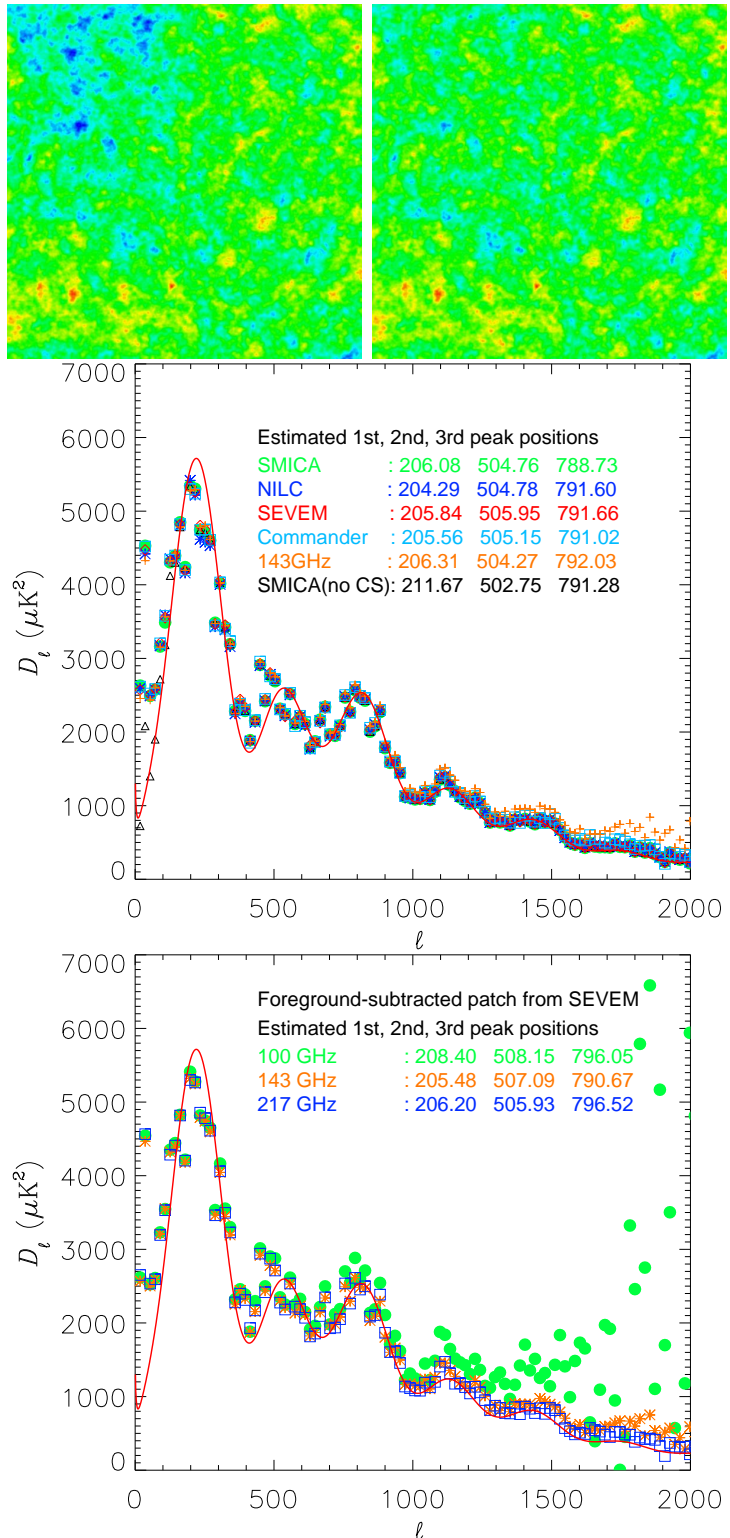

Figure 5. The patch containing the well-known CS from SMICA map (top left panel) and with the CS subtracted (top right), where color bar range is $[-500,500] \mu \mathrm{K}$. Middle panel shows the angular power spectra of the CS patch from Planck 4 maps with green filled circle, blue asterisk, red diamond and light blue square sign denoting SMICA, NILC, SEVEM and Commander patches, respectively. One can see the data points of the first 3 peaks are mostly on the left side of the best-fit $\Lambda$ CDM model (red curve). For comparison, we also show that of the same field from Planck $143 \mathrm{GHz}$ map (orange plus sign), which still contains foreground, and is corrected only for the extra power from non-periodic boundary condition. Even with foreground, the peak positions at $143 \mathrm{GHz}$ are close to those of the CMB patches. Also shown is that of the CS-subtracted square (black triangle). In the bottom panel, we show the same for the CS patch of the foreground-subtracted maps at 100, 143 and $217 \mathrm{GHz}$ from SEVEM method (green filled circle, orange asterisk and blue square, respectively).

smaller $\ell$ s. To see this, we conduct CMB simulations with Planck best-fit $\Lambda \mathrm{CDM}$ model, in total we simulate 4,000 realizations of full-sky CMB maps (with 5arcmin angular resolution). We use spherical Mexican hat wavelet (SMHW) (Martínez-González et al. 2002; Vielva et al. 2004) to analyze the set of data by con- 
volving the SMHW on the whole simulated map via

$$
w(R, p)=\sum_{\ell m} a_{\ell m} W_{\ell}(R) Y_{\ell m}(p),
$$

where $Y_{\ell m}$ is the spherical harmonics, $a_{\ell m}$ is the spherical harmonic coefficients of the simulated map, $p$ is the position/pixel, $W_{\ell}(R)$ is the SMHW window function at scale $R$, and $w(R, p)$ is the SMHW coefficient. We adopt $R=300$ arcmin and found 119 simulated cold spots that have the SMHW coefficients smaller than that of the Planck CS. We further use the CS lowest temperature as the criterion to eliminate 13 , so that the 106 cold spots have deeper dip than the CS (see one example in top left panel of Fig.6). We place the cold spots at the center of $20 \times 20 \mathrm{deg}^{2}$ patches and plot in Fig.6 the normalized histograms of the 3 peak positions and of the distance from the Planck best-fit positions $\left(\ell^{(1)}, \ell^{(2)}, \ell^{(3)}\right)=(220.0,537.5,810.8)$ (Planck Collaboration: Aghanim et al. 2016). For comparison, we plot those from 100 patches from the Planck lensing simulations. We further shift all the cold spots by $10 \mathrm{deg}$ (top right panel as an example) so that roughly half of the cold spot is on the edge of the patch to mimic the CS patch and plot the histograms. One can see the normalized histograms of the peak positions and the distance of the full- and half-cold spot patches do not show any significant skew or shift compared to those from the ordinary CMB patches.

Moreover, extended cold areas with size $>2^{\circ}$ are ubiquitous in $\mathrm{CMB}$, which manifest themselves in the angular power spectrum at $\ell<90$, outside the range of the acoustic peaks. Thus, a more straightforward way to demonstrate the temperature dip in CS itself doesn't cause peak shifts is that we subtract the CS shape from the patch as follows: we take only the upper left quarter of the CS patch, where the CS is located, and set the temperature of the rest of the patch zero. Then it is smoothed with $\sigma=1^{\circ}$, with which the CS patch is subtracted. The patch now is without the prominent dip, shown in the top right panel in Figure 5, and the corresponding power spectrum is shown with black dot in the middle of the same Figure. One can see the CS decrement is mitigated with power spectrum being modified only at $\ell<180$.

To check the significance of the distance of the CS patch, we calculate the distance of the peaks from the 9000 patches from the $100 \mathrm{CMB}$ realizations. In the bottom panel of Figure 4, the black curve is the histogram of the SMICA map and the red 9000 simulated patches. Out of 9000 patches, only 2 with the distance larger than that of the CS patch. If one considers, however, synchronous shift towards smaller multipole numbers, there is none out of 9000 (where the highest distance with all peaks shifting towards large scales is 36.91), pushing the significance to less than $1.11 \times 10^{-4}$. With the CS patch taken on the equator $\phi=\left[180^{\circ}, 200^{\circ}\right]$ of the rotated SMICA map with $\theta=+67.5^{\circ}$, there is another slight shift from the Cold Spot patch taken at the same position from $+66^{\circ}$-rotated map, where the peak distance is 38.4 and shifts towards smaller multipole numbers. That an adjacent area also has the significance less than $1.11 \times 10^{-4}$ shows the CS patch is not a fluke.

Below we discuss possible errors affecting the angular power spectrum. Since we compare the distance of the
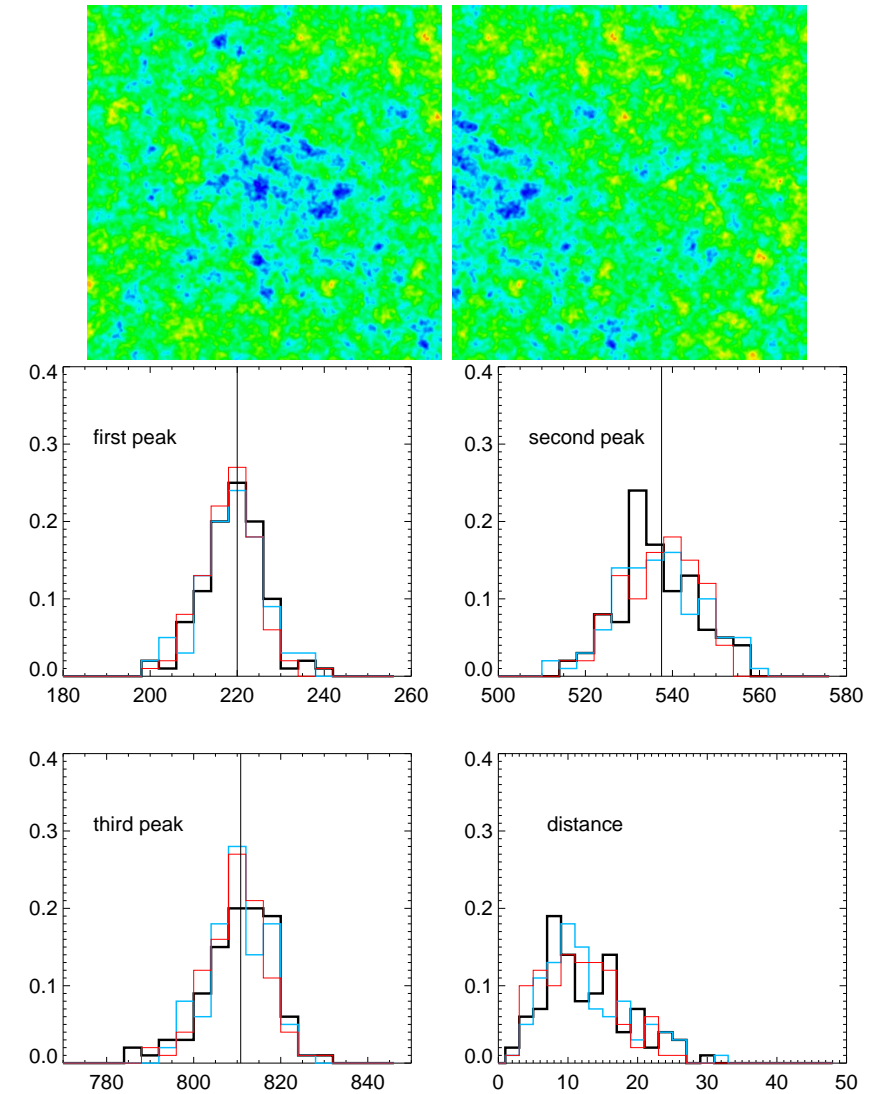

Figure 6. Top left panel is an example of a simulated cold spot placed roughly in the center of a $20 \times 20 \mathrm{deg}^{2}$ patch, whereas the right panel is that by shifting $10 \mathrm{deg}$ such that half of the cold spot is on the edge of the patch to mimic the CS patch Bottom 4 panels show the normalized histograms of the first 3 peak positions and the peak distance from 100 simulated fullcold spots placed at the patch center (black curves) and 100 half-cold spots (blue curves). For comparison, we plot the same for 100 patches taken from the Planck lensing simulation (red curves). The vertical lines denote the Planck best-fit peak positions $\left(\ell^{(1)}, \ell^{(2)}, \ell^{(3)}\right)=(220.0,537.5,810.8)$, respectively. One can see the distributions of peak positions and distance do not display any skew or shift compared to those from the ordinary CMB patches, and the distance from all 3 groups are less than 32 .

Cold Spot patch with those of the simulations including lensing and Doppler boosting effects, one can rule out both effect. The other common culprit is the foreground residual. One should note, however, that the patch is at high Galactic latitude, where the foreground contamination is relatively low. To demonstrate that even the foreground doesn't affect much of the peak positions, we also plot the angular power spectrum of the same field from Planck $143 \mathrm{GHz}$ channel map in middle panel of Figure 5 , which is only corrected for NPB effect. The shoot-up in power is due to the deconvolution on both the foregrounds and noise. One can see the peak positions shift very little even with the presence of the foregrounds, let alone foreground residual after the cleaning. We also plot the power spectra of the Cold Spot patch from the foreground-subtracted maps at 100, 143, $217 \mathrm{GHz}$ from SEVEM method in the bottom panel, and they show the same trend. The summary of the variance of maps and estimated peak positions of the CS patch is in Table 1 .

Even if there is still unknown residual after foreground cleaning, we can use analytical approach to estimate the 


\begin{tabular}{|l||l|}
\hline \multicolumn{2}{|c|}{ Various maps } \\
\hline Map & Variance from 45 patches \\
\hline SMICA & 236.79 \\
NILC & 233.45 \\
SEVEM & 227.37 \\
Commander & 232.07 \\
$143 \mathrm{GHz}$ & 226.56 \\
\hline
\end{tabular}

\begin{tabular}{|l||l|l|l|}
\hline \multicolumn{4}{|c|}{ CS patches from various maps } \\
\hline Patch & 1st peak & 2nd peak & 3rd peak \\
\hline SMICA & 206.08 & 504.76 & 788.73 \\
NILC & 204.29 & 504.78 & 791.60 \\
SEVEM & 205.84 & 505.95 & 791.66 \\
Commander & 205.56 & 505.15 & 791.02 \\
SMICA (no CS) & 211.67 & 502.75 & 791.28 \\
$143 \mathrm{GHz}$ & 206.31 & 504.27 & 792.03 \\
\hline
\end{tabular}

\begin{tabular}{|l||l|l|l|}
\hline \multicolumn{4}{|c|}{ Foreground-subtracted CS Patches from SEVEM method } \\
\hline patch & 1st peak & 2nd peak & 3rd peak \\
\hline $100 \mathrm{GHz}$ & 208.40 & 508.15 & 796.05 \\
$143 \mathrm{GHz}$ & 205.48 & 507.09 & 790.67 \\
$217 \mathrm{GHz}$ & 206.20 & 505.93 & 796.52 \\
\hline
\end{tabular}

Table 1

Top Table shows the variance from the 45 patches of various maps, the middle and bottom tables show the estimated peak positions of the CS patches from various maps.

error budget on the peak positions. We assume any unknown foreground or systematic residual has power-law power spectrum $B \ell^{-\lambda}$, then we can model the residual and an acoustic peak at $\ell_{0}$ with amplitude $A$ and spread $\sigma$ via $D_{\ell}=A \exp \left[-\left(\ell-\ell_{0}\right)^{2} / 2 \sigma^{2}\right]+B \ell(\ell+1) \ell^{-\lambda} \exp [\ell(\ell+$ $\left.1) b^{2}\right] / 2 \pi$, where $b$ is the beam transfer function (see Appendix C). It is easy to see that, for $\lambda<2$, the resultant $D_{\ell}$ at high $\ell$ is dominated by $B \ell^{2-\lambda} \exp \left[\ell(\ell+1) b^{2}\right] / 2 \pi$. In the Planck SMICA, NILC and SEVEM patches, in particularly the patch containing the Cold Spot, the shootup is not seen at high $\ell$, indicating the residual coefficient $B$ has to be extremely small to counter both the deconvolution $\exp \left[\ell(\ell+1) b^{2}\right]$ and $\ell^{2-\lambda}$. Thus peak shift from a small $B$ is negligible. For $\lambda>2$, on the other hand, the peak shift is approximately proportional to $B \sigma^{2} / A \ell_{0}^{\lambda-1}$. The $B$ will have to be much higher than the best-fit $\Lambda \mathrm{CDM}$ model at low $\ell$ to have any significant peak shift, which again is not seen in the angular power spectrum of the CS patch, either. For $\lambda=2$ the peak positions are mostly unchanged because the $\ell(\ell+1)$ cancels out the residual.

\section{DISCUSSION}

Investigation of the CMB CS is well documented. Since its discovery by Vielva et al. (2004), there have been proposals to explain such deviation from Gaussianity: foreground (Cruz et al. 2006; Hansen et al. 2012), multiple voids (Naidoo, Benoit-Lev́y and Lahav 2016), supervoid (Inoue \& Silk 2006, 2007), cosmic texture (Zhao 2013; Cruz et al. 2005), adiabatic perturbation on the last scattering surface (Valkenburg 2012). In particular, a supervoid is found with the size $\sim 220 h^{-1}$ Mpc to explain the lower than usual temperature by Szapudi et al. (2015). It is, however, refuted later that the model of a supervoid alone cannot explain the Cold Spot decrement (Nadathur et al. 2014; Finelli et al. 2016; Mackenzie et al. 2017; Marcos-Caballero et al. 2016). Nevertheless, in trying to justify such model, Szapudi et al. (2015) use WISE-
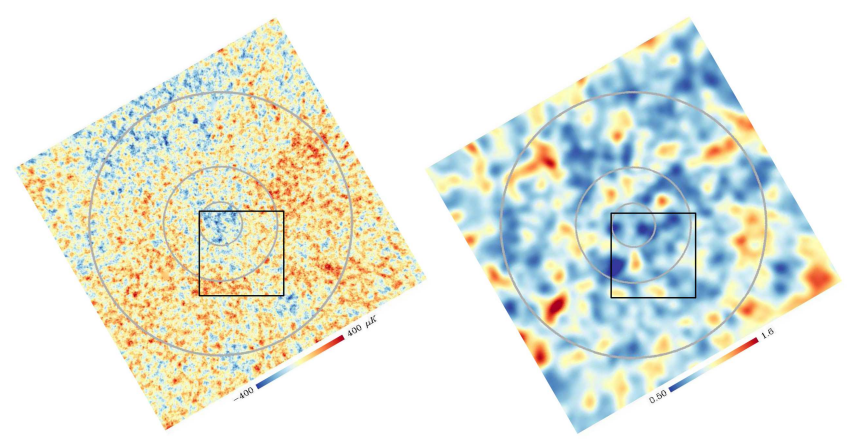

Figure 7. The SMICA field centred at the CS (left) and the corresponding WISE-2MASS field (right), both panels taken from Szapudi et al. (2014) (see also Finelli et al. (2016)). The gray circles from inner to outer correspond to radii of $5^{\circ}, 14^{\circ}$ and $29^{\circ}$, respectively. The black square indicates our CS patch as shown in top left panel of Figure 5. As is stated in Szapudi et al. (2014): "the size of the underdense region is surprisingly large: it is detected to $\sim 20^{\circ}$ with high $(\geq 5 \sigma)$ significance". One can see the surprisingly large underdense region (dark blue region) is mostly included in the square patch.

2MASS catalog to identify underdensities with $z<0.3$, from which a surprisingly large underdense region $\sim 20^{\circ}$ close to the CS with high $(\geq 5 \sigma)$ significance is detected (Szapudi et al. 2014). In Figure 7 we reproduce the figure 2 from Szapudi et al. (2014), and rotate $29^{\circ}$ counterclockwise. The black square indicates exactly the CS patch we are investigating in this paper. One can clearly see that the large underdense region (dark blue) in the right panel is mostly included in the square.

The combination of lower than usual temperature of the CS (significance $\sim 0.01$ ) and larger than usual shifts towards large scales in the acoustic peaks of the patch $\left(1.1 \times 10^{-4}\right)$ results in a $4.73 \sigma$ (significance $p \sim$ $1.11 \times 10^{-6}$ ) observation against the $\Lambda \mathrm{CDM}$ model. With another $5 \sigma$ significance large underdense region in the patch, there are 3 different anomalies in one patch that requires explanation. One should note that voids (either multiple voids or a supervoid) can't shift the acoustic peaks.

Here we propose one of the possible accounts that can explain simultaneously for these anomalies: there is some localized unknown energy in the transverse direction to stretch the space around the CS region. When space is stretched, the wavelength of sinusoidal waves are elongated so that the peaks in the harmonic domain are shifted towards larger scales, and the number density of photons is reduced, while redshifting reduces their frequency, so that the temperature is dragged down. Such stretching of space could also make the density at low redshift lower. If the unknown energy is from dark energy, this implies the existence of inhomogeneity of the dark energy(de Putter, Huterer \& Linder 2010; Blomqvist, Mörtsell \& Nobili 2008; Blomqvist, Enander \& Mörtsell 2010).

This work is supported by ROC MoST grant 1032112-M-001-028. The author acknowledges the use of ESA Planck Legacy Archive data ${ }^{2}$, HEALPIX ${ }^{3}$ package

\footnotetext{
2 http://pla.esac.esa.int/pla/\#home

3 http://healpix.jpl.nasa.gov/
} 
(Górski et al. 2005) and GLESP ${ }^{4}$ package (Doroshkevich et al. 2005). The author would like to thank
D.Spergel, P.Naselsky, A.Lewis, O.Doré and S.Suyu for useful discussions and suggestions, also Ya-Shiuan Lin for helping to produce top panel of Figure 4.

\section{APPENDIX}

\section{NON-PERIODIC BOUNDARY (NPB) CORRECTION FOR PATCHES WITH BEAM CONVOLUTION}

Fourier transform presumes the signal is periodic such that the two ends are continuous, thus when one deals with real data it is inevitable to encounter jump discontinuity, which induces extra power in the power spectrum. Although the data are mostly discrete, the NPB effect nevertheless persists and should be taken into account as systematic error. A patch taken from a full-sky map has such intrinsic effect, which is uncorrectable because NPB is part of the morphology. The effect, however, is further aggravated when there is beam convolution. As the statistics and comparisons in this paper are made between beam-convolved Planck data and simulations with best-fit $\Lambda$ CDM model (without beam convolution), it is necessary to study the effect because the power spectrum of a beam-convolved patch has more induced power than the smoothed power spectrum of the non-periodic patch.

To estimate the correction, we simulate 50 full-sky CMB maps and take $30 \mathrm{deg}^{2}$ patches centered at the equator (in total 600 patches). We take the central $20 \mathrm{deg}^{2}$ part directly from the $30 \mathrm{deg}^{2}$ patches, and denote their mean power spectrum as $C_{\ell}^{A}$. Then we use 5 arcmin to convolve the $30 \mathrm{deg}^{2}$ patches and choose the central $20 \mathrm{deg}^{2} \mathrm{part}_{\text {, the mean }}$ power spectrum of which is denoted $C_{\ell}^{B}$. The correction is therefore $C_{\ell}^{B}-b^{2} C_{\ell}^{A}$, where $b$ is the beam transfer function. One finds that the extra power due to 5 -arcmin beam convolution is a rather smooth curve and can be best-fitted to be $C_{\ell}^{\mathrm{NPB}}=1.21 \ell^{-1.38}\left(\mu \mathrm{K}^{2}\right)$ from $\ell \geq 300$. And for $143 \mathrm{GHz}$ band map with beam transfer function 7.30 arcmin (Planck Collaboration: Adam et al. 2016a) the correction for NPB is $C_{\ell}^{\mathrm{NPB}}=34.4 \ell^{-1.80}\left(\mu \mathrm{K}^{2}\right)$.

\section{CROSS-POWER SPECTRUM ON THE Planck DATA}

Throughout the paper we use cross-power spectrum (XPS) on the Planck data : 4 CMB maps, $143 \mathrm{GHz}$ channel map, and the foreground-subtracted maps at 100, 143, and $217 \mathrm{GHz}$ from the SEVEM method, to extract the peak position. The XPS is a quadratic estimator between two maps that can provide unbiased estimate of the underlying power spectrum of the correlated signals, and, at the same time, reduce the uncorrelated ones. We take from Planck Legacy Archive the FULL MISSION RINGHALF1 and FULL MISSION RINGHALF2 maps for SMICA, NILC, SEVEM, Commander and 143GHz map. Since the patches from RINGHALF1 (denoted $\alpha$ ) and RINGHALF2 (denoted $\beta$ ) contain the same CMB signal but uncorrelated noise, we can employ the XPS on their Fourier modes $\alpha_{\boldsymbol{k}}$ and $\beta_{\boldsymbol{k}}$

$$
S_{k}^{\alpha \beta}=\frac{1}{2}\left\langle\alpha_{\boldsymbol{k}}^{*} \boldsymbol{k}+\beta_{\boldsymbol{k}}^{*} \alpha_{\boldsymbol{k}}\right\rangle,
$$

where $*$ denotes complex conjugate and the angle brackets denote average over all $k-1 / 2 \leq|\boldsymbol{k}|<k+1 / 2$ for integer k. However, as Chiang \& Chen $(2011,2012)$ pointed out, lack of an ensemble and non-zero chance correlation between the so-called "uncorrelated" signal results in some residual $X_{k}^{\alpha \beta}$ from the XPS, which has the following relation,

$$
\sqrt{\left\langle\left(X_{k}^{\alpha \beta}\right)^{2}\right\rangle} \simeq \frac{\sqrt{N_{k}^{\alpha} N_{k}^{\beta}}}{\sqrt{2 \pi k}},
$$

where $N_{k}^{\alpha}$ and $N_{k}^{\beta}$ are the (uncorrelated) noise power spectrum from RINGHALF1 and RINGHALF2, respectively. The noise power spectrum can be obtain by $1 / 2$ of the differencing of HALFRING1 and HALFRING2 patches, assuming $N_{k}^{\alpha}$ and $N_{k}^{\beta}$ are of the same level. Thus in our data pipeline, we subtract the residual $X_{k}^{\alpha \beta}$ from the XPS, after the correction of NPB.

\section{FOREGROUND RESIDUAL ESTIMATE}

The foreground residual (or any unknown residual or systematics) can take the form of $B \ell^{-\lambda}$ after foreground cleaning such that we can model the residual and an acoustic peak at $\ell_{0}$ with amplitude $A$ and spread $\sigma$ by $D_{\ell}=$ $A \exp \left[-\left(\ell-\ell_{0}\right)^{2} / 2 \sigma^{2}\right]+B \ell(\ell+1) \ell^{-\lambda} \exp \left[\ell(\ell+1) b^{2}\right] / 2 \pi$, where $b$ is the beam transfer function. As deconvolution of the beam transfer function varies slowly (for the multipole range in our discussion) we assume $\exp \left[\ell(\ell+1) b^{2}\right] \simeq w$. Letting $\beta \equiv \pi A / \sigma^{2}$ and $\epsilon \equiv B / \beta$, one can solve for the peak position for $\lambda=0,1,2: \ell \simeq \ell_{0}(1-\epsilon w)^{-1}, \ell \simeq \ell_{0}+\epsilon w / 2$, and $\ell \simeq \ell_{0}$, respectively. Further assuming the peak position not far from $\ell_{0}$ for $\lambda=3, \ell \sim \ell_{0}-\epsilon w / 2 \ell_{0}^{2}$.

For $\lambda=0$ the peak position is shifted by $\ell_{0} \epsilon w(1-\epsilon w)^{-1}$, which could be large enough to be the source of the anomaly. One should note, however, that if there is any residual with $\lambda=0$ (flat spectrum), it should be mixed with the pixel noise and is duly subtracted in our data processing. For $\lambda=1$, the peak shift $\epsilon w / 2$ is inversely proportional to $\beta$, where $\beta$ is $1.79,0.576,0.446$ for 1 st, 2 nd and 3 rd peak of best-fit $\Lambda$ CDM model, respectively. For the shift to be significant, $B$ has to be of the same order of $\beta$. Such high level of residual $B$, together with $(\ell+1) \exp \left[\ell(\ell+1) b^{2}\right]$ would have caused the power to shoot up at high $\ell$, but in reality the power spectrum of the Cold Spot patch in the

${ }^{4}$ http://www.glesp.nbi.dk/ 
middle panel of Figure 5 shows no such shoot-up at all. For $\lambda=2$ the peak position is mostly static as the $\ell(\ell+1)$ cancels out the residual.

The case of $\lambda=3(\lambda>2)$ is different from the previous cases in that the power index of the residual part in $D_{\ell}$ is negative $\left(\sim \ell^{-1}\right)$. The peak shift, inversely proportional to $\ell_{0}^{2}$, is less than unity even when $B$ is as high as 9000 , making the residual at the same level of best-fit $\Lambda$ CDM model quadrupole.

Although the foreground residual is unlikely to have power law with integer index, the analytical forms display the following trend: for $\lambda<2$ the resultant $D_{\ell}$ is dominated by $B \ell^{2-\lambda} \exp \left[\ell(\ell+1) b^{2}\right]$ at high $\ell$. In the Planck data, in particularly the patch containing the Cold Spot, the shoot-up is not seen at high $\ell$, indicating the residual coefficient $B$ has to be quite small to counter the deconvolution and $\ell^{2-\lambda}$. The peak shift is thus negligible. For $\lambda>2$, on the other hand, the peak shift is approximately proportional to $\epsilon w / \ell_{0}^{\lambda-1}$. The $B$ will have to be much higher than the best-fit $\Lambda$ CDM model at low $\ell$ to have any significant peak shift, which again is not seen in the power spectrum in the middle of Figure 5, either.

\section{REFERENCES}

de Bernardis, P., et al., 2000, Nature, 404, 955

Blanchard, A., Schneider, J., 1987, A\&A, 184, 1

Blomqvist, M., Mörtsell, E., Nobili, S., 2008, J. Cosmo. Astropar., 6, 27

Blomqvist, M., Enander, J., Mörtsell, E., 2010, J. Cosmo. Astropar., 10, 18

Challinor, A., Lewis, A., 2002, Phys. Rev. D, 65, 103001

Challinor, A., Lewis, A., 2005, Phys. Rev. D, 71, 103010

Chiang, L.-Y., Chen, F.-F., 2011, ApJ, 738, 188

Chiang, L.-Y., Chen, F.-F., 2012, ApJ, 751, 43

Cole, S., Efstathiou, G., 1989, MNRAS, 239, 195

Cruz, M., Martínez-González, E., Vielva, P., Cayón, L., 2005, MNRAS, 356, 29

Cruz, M., Tucci, M., Martínez-González, E., Vielva, P., 2006, MNRAS, 369, 57

de Putter, R., Huterer, D., Linder, E.V., 2010, Phys. Rev. D, 81, 103513

Doroshkevich, A.G., et al., 2005, Int. J. Mod. Phys. D, 14, 275

Finelli, F., et al., 2016, MNRAS, 455, 1246

Górski, K.M., et al., 2005, ApJ, 622, 759

Halverson, N.W., et al., 2002, ApJ, 568, 38

Hanany, S., et al., 2000, ApJ, 545, L5

Hansen, M., et al., 2012, MNRAS, 426, 57

Hinshaw, G., et al., 2007, ApJS, 170, 288

Hinshaw, G., et al., 2013, ApJS, 208, 19

Hu, W., Dodelson, S., 2002, ARA\&A, 40, 171

Inoue, K.T., Silk, J., 2006, ApJ, 648, 23

Inoue, K.T., Silk, J., 2007, ApJ, 664, 650
Lewis, A., Challinor, A., 2006, Phys. Rep., 429, 1

Linder, E.V., 1990, ApJ, 243, 353

Jeong, D., et al., 2014, Phys. Rev. D, 89, 023003

Mackenzie, R., et al., 2017, MNRAS, 470, 2328

Marcos-Caballero, A., Fernández-Cobos, R., Martínez-González, E., Vielva, P., 2016, MNRAS, 460, L15

Martínez-González, E., et al., 2002, MNRAS, 336, 22

Nadathur, S., et al., 2014, Phys. Rev. D, 90, 103510

Naidoo, K., Benoit-Lev́y, A., Lahav, O., 2016, MNRAS, 459, L71

Planck Collaboratoin: Ade P.A.R. et al., 2014a, A\&A, 571, A15

Planck Collaboratoin: Ade P.A.R. et al., 2014b, A\&A, 571, A12

Planck Collaboratoin: Ade P.A.R. et al., 2014c, A\&A, 571, A17

Planck Collaboratoin: Aghanim N. et al., 2014, A\&A, 571, A27

Planck Collaboratoin: Adam R. et al., 2016a, A\&A, 594, A7

Planck Collaboratoin: Adam R. et al., 2016b, A\&A, 594, A9

Planck Collaboratoin: Aghanim N. et al., 2016, A\&A, 594, A11

Planck Collaboratoin: Ade P.A.R. et al., 2016a, A\&A, 594, A15

Planck Collaboratoin: Ade P.A.R. et al., 2016b, A\&A, 594, A16

Press, W.H., et al., 1994, Numerical Recipes in Fortran,

Cambridge University Press, 2nd Ed.

Seljak, U., 1996, ApJ, 463, 1

Scott, P.F., et al., 2003, MNRAS, 341, 1076

Szapudi, I., et al., 2014, Proceedings of 49th Rencontres de Moriond Conference Cosmology 2014, edited by E. Aug, J.

Dumarchez and J. Tran Thanh Van, 33

Szapudi, I., et al., 2015, MNRAS, 450, 288

Valkenburg, W., 2012, J. Cosmo. Astropar., 01, 47

Vielva, P., et al., 2004, ApJ, 609, 22

Zhao, W., 2013, MNRAS, 433, 3498 